\title{
TWO-DIMENSIONAL INHOMOGENEITIES OF MINIMUM STRESS CONCENTRATION*
}

BY

\author{
REN - JIEH SHIH AND LEWIS T. WHEELER \\ Unitersity of Houston--University Park
}

1. Introduction. The object of this paper is a two-dimensional optimization problem concerning the best shape for an inhomogeneity in an elastic matrix of unbounded extent, in which the shape is to be chosen so as to minimize the stress concentration in the matrix. The inhomogeneity takes the form of a rigid inclusion or a cavity whose walls are subjected to uniform pressure. The response of the matrix, which deforms under the influence of remotely applied stress, is governed by the linear theory for homogeneous and isotropic elastic solids in states of plane deformation. In the case of a rigid inclusion, the inhomogeneity is free of external force. In other words, the only forces acting on the inclusion are the tractions acting along the bond with the matrix. These boundary value problems are formulated in Sec. 2.

Though the response of the matrix is governed by a linear system of partial differential equations, the optimization problem under consideration is nonlinear owing to the fact that variations are performed on the geometry. This optimization problem is formulated in Sec. 3, at which stage it becomes essential to define precisely the manner in which stress concentration is measured. This brings us to the main point of the present paper. Unlike previous work, which is concerned with materials that fail in accordance with the von Mises criterion [1,2] or involve the "Euclidean" stress norm [3-8], we here treat materials that fail in accordance with the maximum principal stress theory for brittle solids.

The strategy for solving the optimization is to reduce it to an "inverse problem," a strategy which has met with considerable success [9-18], although the earlier work [9-11] did not make specific reference to this notion. The form of this problem appropriate to the present research is described in Sec. 4 and solved in Sec. 5. We should mention that this version of the inverse problem has been dealt with in several previous investigations in which its relation to optimization problems similar to those of the present paper was described with varying degrees of exactness. In the present paper, as in $[1-6,8]$, the reducibility of the optimization problem to an inverse problem is proved mathematically.

\footnotetext{
* Received July 26, 1985.
} 
Although the inverse problem has been dealt with many times previously, we believe it worthwhile to include here a section dealing with its solution because the previous work requires recourse to complex variable methods which are, of course, quite powerful but typically leave the results in a form that is not particularly transparent. Here we include a derivation, which, though it may lack the generality of the complex variable approach, is somewhat more elementary and direct. The resulting expressions for the elastic fields associated with the solution of the inverse problem are expressed in terms of the logarithmic potential of a homogeneous ellipse. While such expressions must, of course, follow from the derivations based upon complex variables, we have not encountered them in the literature.

In Sec. 6, the final section of the paper, we close the connection between the inverse and optimization problems by particularizing the solution of the inverse problem to the cases under consideration here, namely, voids and rigid inclusions. We thus arrive at results describing the optimum shapes for the inhomogeneities, which assume the form of suitably proportioned ellipses. Illustrations are given for the stress fields corresponding to a rigid inclusion, a traction-free cavity, and a pressurized cavity.

2. Formulation of the two-dimensional boundary-value problems for an elastic solid with an inhomogeneity. Let $D$ denote an exterior plane domain formed by the deletion of a bounded region, and denote by $C$ the boundary of $D$. Occupying $D$ is a homogeneous and isotropic elastic medium that assumes a state of plane strain under the influence of stresses applied at infinity. Body force is absent.

For the plane states of deformation presently under consideration, the in-plane components of displacement, strain, and stress, $u_{\alpha}, \varepsilon_{\alpha \beta}, \tau_{\alpha \beta}$, respectively, are governed by the field equations ${ }^{1}$

$$
\begin{gathered}
\varepsilon_{\alpha \beta}=\frac{1}{2}\left(u_{\alpha, \beta}+u_{\beta, \alpha}\right), \\
\varepsilon_{\alpha \beta}=(1+\nu)\left(\tau_{\alpha \beta}-\nu \tau_{\gamma \gamma} \delta_{\alpha \beta}\right) / E \\
\tau_{\alpha \beta, \beta}=0
\end{gathered}
$$

where $\nu$ denotes Poisson's ratio, and $E$ Young's modulus. As for conditions at infinity,

$$
\tau_{\alpha \beta} \rightarrow \stackrel{\infty}{\tau}_{\alpha \beta} \quad \text { as }|x| \rightarrow \infty, \quad \stackrel{\infty}{\tau}_{\alpha \beta}=\sum_{\gamma=1}^{2} \stackrel{\infty}{\tau}_{\gamma} \delta_{\alpha \gamma} \delta_{\beta \gamma} .
$$

In the present paper, we consider two cases: within the region complementary to $D$, there lies a rigid inclusion or a void whose walls are uniformly pressurized. For the case of the void, the boundary conditions require the traction components $t_{\alpha}$ to obey

$$
t_{\alpha}=\tau_{\alpha \beta} n_{\beta}=-P n_{\alpha} \text { on } C,
$$

\footnotetext{
${ }^{1}$ Greek letter subscripts have the range $\{1,2\}$; subscripts preceded by a comma indicate partial differentiation with respect to the corresponding coordinates.
} 
where $P$ is a given positive constant and $n_{\alpha}$ denotes the components of the unit normal to $C$, directed outward from $D$. On the other hand, for a rigid inclusion, the requirement is that the displacements $u_{\alpha}$ be of the form

$$
u_{\alpha}=a_{\alpha}+b e_{\alpha \beta} x_{\beta} \quad \text { on } C, \quad e_{\alpha \beta}= \begin{cases}1 & \text { if }(\alpha, \beta)=(1,2), \\ 0 & \text { if }(\alpha, \beta)=(1,1) \text { for }(\alpha, \beta)=(2,2), \\ -1 & \text { if }(\alpha, \beta)=(2,1)\end{cases}
$$

where $a_{\alpha}$ and $b$ are constants, which are not prescribed, but must be found by solving the boundary value problem. The inclusion itself is assumed to be free of force, and as a consequence, $t$ must obey

$$
\int_{C} \mathbf{t} d s=0, \quad \int_{C} \mathbf{x} \times \mathbf{t} d s=0 .
$$

We introduce stress components $\tau_{N}$ and $\tau_{S}$ through

$$
\tau_{N}=\tau_{\alpha \beta} n_{\alpha} n_{\beta}, \quad \tau_{S}=\tau_{\alpha \beta} s_{\alpha} s_{\beta},
$$

where

$$
s_{\alpha}=e_{\alpha \beta} n_{\beta},
$$

so that $\tau_{N}$ stands for the normal stress acting on $C$ and $\tau_{S}$ the stress acting tangentially in the surface described by $C\left(\tau_{S}\right.$ is thus the "hoop" stress.). Because $\tau_{\alpha \alpha}=\theta$ is invariant under changes in the underlying Cartesian coordinate frame, it follows that

$$
\tau_{N}=-P, \quad \tau_{S}=\theta+P
$$

in the case of the void. Finally, we note that for the case of the rigid inclusion it is not difficult to show (see [1]) with the aid of (2.1), (2.2), and (2.6) that

$$
\tau_{N}=(1-\nu) \theta, \quad \tau_{S}=\nu \theta .
$$

3. Formulation of the optimization problem and inverse problem. Let $\tau_{1}$ and $\tau_{2}$ denote the principal stresses in $D$, and let these be ordered so that

$$
\tau_{1} \leqslant \tau_{2} \text { on } D \text {. }
$$

According to the maximum principal stress theory (also known as the Rankine criterion [19]), the material is on the verge of failure if

$$
\tau_{2}=\tau_{\mathrm{cr}},
$$

where $\tau_{\mathrm{cr}}$ denotes the tensile strength of the material. Let

$$
\tau_{2}^{M}[D]=\sup _{D} \tau_{2}
$$

Then the optimization problem that we are considering is to find a region $D^{*}$ on which the greatest value of the principal stress $\tau_{2}$ is minimum, or

$$
\inf \tau_{2}^{M}[D]=\tau_{2}^{M}\left[D^{*}\right] \stackrel{\text { def }}{=} \tau_{2}^{m},
$$


where the infimum is taken over the class of all exterior regions bounded internally by a single closed curve. The region $D^{*}$ is said to be optimal (with respect to the maximum principal stress criterion) if (3.4) holds.

Our approach to the solution of the optimization problem is to reduce it to an inverse problem, by which we mean that given $\stackrel{\infty}{\tau}_{\alpha}, P$, and $\nu$, find $C^{*}$ such that on $C^{*}$ the corresponding stress components $\tau_{\alpha \beta}^{*}$ are of the form

$$
\tau_{\alpha \beta}^{*}=S\left(\delta_{\alpha \beta}-n_{\alpha} n_{\beta}\right)-P n_{\alpha} n_{\beta} \quad \text { on } C^{*},
$$

where $S$ is a constant.

In the next section, we give a rigorous proof that such a reduction is permissible.

4. Transformation of the optimization problem. In the following theorem, we summarize certain properties of the solution of the inverse problem that will play a key role in showing how it can be used to solve the underlying optimization problem.

Theorem 1. Assume that on $C^{*}, \tau_{\alpha \beta}^{*}$ is of the form

$$
\tau_{\alpha \beta}^{*}=S\left(\delta_{\alpha \beta}-n_{\alpha} n_{\beta}\right)-P n_{\alpha} n_{\beta},
$$

where $S$ and $P$ are constants. Then

(a) $\tau_{\gamma \gamma}^{*}=\stackrel{\infty}{\tau}_{\gamma \gamma}=S-P$ on $D^{*}$,

(b) $\Delta \tau_{\alpha \beta}^{*}=0$ on $D^{*}$,

(c) $\tau_{2}^{*}=\max \{-P, S\}$ on $C^{*}$,

(d) $\tau_{2}^{*} \leqslant \max \left\{\stackrel{\infty}{\tau}_{2},-P, S\right\}$ on $D^{*}$.

Proof. We note first that (c) follows from the fact that $-P$ and $S$ are principal stresses. Next, since

$$
\theta^{*}=\tau_{\gamma \gamma}^{*}
$$

is a solution of the Laplace equation, $\theta^{*}$ assumes the constant value

$$
\theta^{*}=S-P \text { on } C,
$$

and is bounded at infinity; it follows that (a) is true. As for (b), it is easy to use the result just established and equations (2.1)-(2.3) to show that $\tau_{\alpha \beta}^{*}$ satisfy Laplace's equation.

Considering now (d), we have

$$
\left(\tau_{2}^{*}-\tau_{1}^{*}\right)^{2}=2\left[\left(\tau_{1}^{*}\right)^{2}+\left(\tau_{2}^{*}\right)^{2}\right]-\left(\tau_{1}^{*}+\tau_{2}^{*}\right)^{2}=2 \tau_{\alpha \beta}^{*} \tau_{\alpha \beta}^{*}-\left(\stackrel{\infty}{\tau_{\gamma \gamma}}\right)^{2} .
$$

Thus,

$$
\Delta\left(\tau_{2}^{*}-\tau_{1}^{*}\right)^{2}=2 \Delta\left(\tau_{\alpha \beta}^{*} \tau_{\alpha \beta}^{*}\right)
$$

which yields

$$
\Delta\left(\tau_{2}^{*}-\tau_{1}^{*}\right)^{2}=4\left(\tau_{\alpha \beta, \gamma}^{*} \tau_{\alpha \beta, \gamma}^{*}+\tau_{\alpha \beta}^{*} \Delta \tau_{\alpha \beta}^{*}\right) .
$$

Therefore, and in view of (b), $\left(\tau_{2}^{*}-\tau_{1}^{*}\right)^{2}$ is a subharmonic function. Hence, and by (3.1),

$$
\tau_{2}^{*}-\tau_{1}^{*}=\left|\tau_{2}^{*}-\tau_{1}^{*}\right| \leqslant \max \left\{\begin{array}{l}
\infty \\
\tau_{2}
\end{array}-\stackrel{\infty}{\tau_{1}},|S+P|\right\} \text { on } D^{*},
$$


Further, we know that

$$
\tau_{1}^{*}+\tau_{2}^{*}=\stackrel{\infty}{\tau_{1}}+\stackrel{\infty}{\tau_{2}} \text { on } D
$$

Thus, (4.5) furnishes

$$
2 \tau_{2}^{*}-\left(\begin{array}{l}
\infty \\
\tau_{1}
\end{array}+\stackrel{\infty}{\tau_{2}}\right) \leqslant \max \left\{\begin{array}{l}
\infty \\
\tau_{2}
\end{array}-\stackrel{\infty}{\tau_{1}},|S+P|\right\} \quad \text { on } D^{*} .
$$

If $\stackrel{\infty}{\tau_{2}}-\stackrel{\infty}{\tau}_{1} \geqslant|S+P|$, we have

$$
2 \tau_{2}^{*} \leqslant\left(\begin{array}{l}
\infty \\
\tau_{1}
\end{array}+\stackrel{\infty}{\tau_{2}}\right)+\stackrel{\infty}{\tau_{2}}-\stackrel{\infty}{\tau_{1}}=2 \stackrel{\infty}{\tau_{2}},
$$

which yields the desired conclusion (d). If $|S+P| \geqslant \stackrel{\infty}{\tau_{2}}-\stackrel{\infty}{\tau}$, we have by (a)

$$
2 \tau_{2}^{*} \leqslant|S+P|-P+S \text { on } D^{*} \text {. }
$$

For the case $-P \geqslant S$, we get

$$
2 \tau_{2}^{*} \leqslant-P-S-P+S=-2 P \text { on } D^{*},
$$

whereas, for $S \geqslant-P$, we have

$$
2 \tau_{2}^{*} \leqslant S+P-P+S=2 S \text { on } D^{*} .
$$

In the following theorem, we establish the connection between the inverse problem and the optimization problem for the case of a void with uniformly pressurized walls.

Theorem 2. Assume that ${ }_{\alpha \beta} n_{\beta}=-P$ on $C$, where $P$ is a nonnegative constant, and also that

$$
\stackrel{\infty}{\tau_{1}} \geqslant 0 \text { and } \quad \stackrel{\infty}{\tau_{1}}+\stackrel{\infty}{\tau_{2}}+P \geqslant 0
$$

Then

$$
\tau_{2}^{M}[D] \geqslant \sup _{D^{*}} \tau_{2}^{*}=\tau_{2}^{m}=\stackrel{\infty}{\tau}_{1}+\stackrel{\infty}{\tau}_{2}+P,
$$

i.e., the solution to the inverse problem is also the solution to the optimization problem.

Proof. At points of $C$,

$$
\tau_{2}=\max \{\theta+P,-P\} .
$$

Thus,

$$
\sup _{D} \tau_{2} \geqslant \sup _{C} \tau_{2}=\max \left\{\sup _{C} \theta+P,-P\right\} \geqslant \sup _{C} \theta+P .
$$

But, by the maximum principle,

$$
\sup _{C} \theta \geqslant \lim _{|x| \rightarrow \infty} \theta=\theta^{*} .
$$

From part (a) of Theorem 1, we deduce that

$$
S=\theta^{*}+P \text { on } D^{*},
$$

while part (d), the assumption that $P \geqslant 0$, and (4.12) yield

$$
\tau_{2}^{*} \leqslant S \text { on } D^{*} \text {. }
$$

Accordingly, and by (4.13) and (4.14) the proof is now complete. 
THEOREM 3. Assume that $C$ encloses a rigid inclusion and that $(1-\nu) \stackrel{\infty}{\tau_{1}} \geqslant \nu \stackrel{\infty}{\nu} \tau_{2} \geqslant 0$. Then

$$
\sup _{D} \tau_{2} \geqslant \sup _{D^{*}} \tau_{2}^{*}=(1-\nu)\left(\begin{array}{l}
\infty \\
\tau_{1}
\end{array}+\stackrel{\infty}{\tau_{2}}\right)
$$

Proof. We have

$$
\sup _{D} \tau_{2} \geqslant \sup _{C} \tau_{2}=(1-\nu) \sup _{C} \theta \geqslant(1-\nu) \theta^{*} .
$$

By Theorem 1, and since

$$
(1-\nu)\left(\begin{array}{ll}
\infty \\
\tau_{1}
\end{array}+\stackrel{\infty}{\tau}_{2}\right) \geqslant \stackrel{\infty}{\tau_{2}}
$$

we have

$$
(1-\nu) \theta^{*}=\sup _{D^{*}} \tau_{2}^{*}
$$

This completes the proof.

5. Solution of the inverse problem. In this section, we demonstrate that a certain class of elliptic cylinders (in two dimensions, ellipses) furnishes a solution of the inverse problem. The logarithmic potential of a homogeneous cylinder of unit mass density is introduced and used to derive explicit expressions for the stresses, strains, and displacements associated with the inverse problem. Alternative approaches based upon complex variable methods are available [9-16]. While the use of complex variables has certain attractive features, as we shall see it is possible to proceed along more direct and elementary lines of reasoning to arrive at fully explicit expressions for the fields presently of interest. Although the results presented here can, of course, be deduced with the aid of the methods of [9-16], our results evidently are the first of their kind to be presented in the literature.

It is assumed that the intersection of the elliptic cylinder with the $x_{1}-x_{2}$ plane forms the ellipse

$$
x_{1}^{2} / a_{1}^{2}+x_{2}^{2} / a_{2}^{2} \leqslant 1 .
$$

The potential $\phi$ of the homogeneous ellipse may be put in the form [20-21]

$$
\begin{aligned}
\phi=2 & \pi a_{1} a_{2}\left\{\frac{1}{2} \int_{k}^{\infty} \frac{d s}{\left[\left(a_{1}^{2}+s\right)\left(a_{2}^{2}+s\right)\right]^{1 / 2}}\right. \\
& \left.\frac{x_{1}^{2}}{a_{1}^{2}+k+\left[\left(a_{1}^{2}+k\right)\left(a_{2}^{2}+k\right)\right]^{1 / 2}}-\frac{x_{2}^{2}}{a_{2}^{2}+k+\left[\left(a_{1}^{2}+k\right)\left(a_{2}^{2}+k\right)\right]^{1 / 2}}\right\}
\end{aligned}
$$

where $k$ is the largest nonnegative root of the equation

$$
x_{1}^{2} /\left(a_{1}^{2}+k\right)+x_{2}^{2} /\left(a_{2}^{2}+k\right)=1 .
$$

As $k$ varies, (5.3) describes a family of confocal ellipses, and for $k=0$ it yields the ellipse which forms the boundary of the elastic solid under consideration. 
As we shall see, the function $\phi$ will serve as a stress function. For the present, we are concerned with certain properties of $\phi$. First of all, $\phi$ satisfies the following equations:

$$
\begin{aligned}
& \phi,{ }_{\alpha \alpha}=0 \text { on } D, \\
& \phi,{ }_{\alpha \alpha}=-4 \pi \text { for } \frac{x_{1}^{2}}{a_{1}^{2}}+\frac{x_{2}^{2}}{a_{2}^{2}}<1 .
\end{aligned}
$$

The derivatives of $\phi$ may be found by differentiating (5.2),

$$
\begin{gathered}
\phi, \alpha=\frac{-4 \pi a_{1} a_{2} x_{\alpha}}{a_{\alpha}^{2}+k+\left[\left(a_{1}^{2}+k\right)\left(a_{2}^{2}+k\right)\right]^{1 / 2}}, \\
\phi, \alpha \beta=-4 \pi a_{1} a_{2}\left\{\frac{-n_{\alpha} n_{\beta}}{\left[\left(a_{1}^{2}+k\right)\left(a_{2}^{2}+k\right)\right]^{1 / 2}}+R_{1} \delta_{1 \alpha} \delta_{1 \beta}+R_{2} \delta_{2 \alpha} \delta_{2 \beta}\right\},
\end{gathered}
$$

where $n_{\alpha}$ are components of the unit normal vector directed outward from the confocal ellipses defined in (5.3), and

$$
R_{\alpha}=\frac{1}{\left(a_{\alpha}^{2}+k\right)^{1 / 2}\left[\left(a_{1}^{2}+k\right)^{1 / 2}+\left(a_{2}^{2}+k\right)^{1 / 2}\right]} .
$$

By inspecting (5.3), it can be shown that if $a_{1}>a_{2}$,

$$
|\mathbf{x}|^{2}-a_{1}^{2}<k<|\mathbf{x}|^{2}-a_{2}^{2} \text {. }
$$

The stress field in our problem can be assumed in the form

$$
\tau_{\alpha \beta}^{*}=\frac{-A}{4 \pi} \phi,_{\alpha \beta}+B \stackrel{\infty}{\tau}_{\alpha \beta},
$$

where $A$ and $B$ are constants to be determined. We observe that because (5.4) holds and $\stackrel{\infty}{\tau \beta}_{\alpha \beta}$ are constants, (2.3) is automatically satisfied. Also since $\phi,,_{\alpha \beta} \rightarrow 0$, the condition $\tau_{\alpha \beta}^{*} \rightarrow \stackrel{\infty}{\tau} \tau_{\alpha \beta}$ as $|\mathbf{x}| \rightarrow \infty$ is satisfied by taking $B=1$. Thus, and by (5.7) and (2.4), it follows that

$$
\tau_{\alpha \beta}^{*}=-A\left\{\frac{a_{1} a_{2} n_{\alpha} n_{\beta}}{\left[\left(a_{1}^{2}+k\right)\left(a_{2}^{2}+k\right)\right]^{1 / 2}}\right\}+\sum_{\gamma=1}^{2}\left(A a_{1} a_{2} R_{\gamma}+\stackrel{\infty}{\tau}_{\gamma}^{\infty}\right) \delta_{\gamma \alpha} \delta_{\gamma \beta},
$$

By substituting $k=0$ into (5.11) and comparing with (3.5), we arrive at

$$
A=S+P \text {, }
$$

and

$$
a_{1} / a_{2}=\left(S-\stackrel{\infty}{\tau}_{2}\right) /\left(S-\stackrel{\infty}{\tau_{1}}\right)
$$

where $S$ and $P$ are determined in (2.10)-(2.11). Thus, the semiaxis ratio can be found

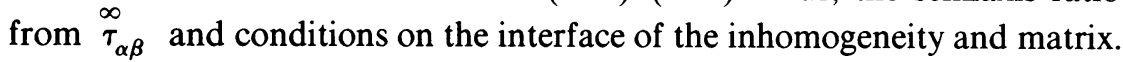

The strain field can be derived by substituting (5.11) and (5.12) into (2.2)

$$
\varepsilon_{\alpha \beta}^{*}=u_{(\alpha, \beta)}=-\frac{P+S}{4 \pi} \frac{1+\nu}{E} \phi,_{\alpha \beta}+\stackrel{\infty}{\varepsilon_{\alpha \beta}},
$$

where

$$
\stackrel{\infty}{\varepsilon}_{\alpha \beta}=(1+\nu)\left(\stackrel{\infty}{\tau}_{\alpha \beta}-\nu \stackrel{\infty}{\tau}_{\gamma \gamma} \delta_{\alpha \beta}\right) / E
$$


Equation (5.14) suggests the following form for the displacement field

$$
u_{\alpha}^{*}=\left[-\frac{P+S}{4 \pi} \frac{1+\nu}{E} \phi+\frac{1}{2} \stackrel{\infty}{\varepsilon}_{\beta \gamma} x_{\beta} x_{\gamma}\right], \alpha,
$$

which can be readily verified.

6. Discussion. It is evident from (5.13) that the analysis of the preceding section does not yield a solution if the right member is negative. Indeed, there is no reason to expect a solution to exist in any form if $S, \stackrel{\infty}{\tau_{1}}$, and $\stackrel{\infty}{\tau}_{2}$ result in a negative right side. For the case of a pressurized void, (2.10) and (5.13) furnish

$$
\frac{a_{1}}{a_{2}}=\frac{S-\stackrel{\infty}{\tau_{2}}}{S-\stackrel{\infty}{\tau}_{1}}=\frac{\stackrel{\infty}{\tau_{1}}+P}{\stackrel{\infty}{\tau}_{2}+P}
$$

whereas for a rigid inclusion, (2.11) and (5.15) yield

$$
\frac{a_{1}}{a_{2}}=\frac{S-\stackrel{\infty}{\tau},}{S-\stackrel{\infty}{\tau_{1}}}=\frac{(1-\nu) \stackrel{\infty}{\tau_{2}}-\nu \stackrel{\infty}{\tau} \tau_{1}}{(1-\nu) \stackrel{\infty}{\tau_{1}-\nu} \stackrel{\infty}{\tau}_{2}}=\frac{\stackrel{\infty}{\varepsilon_{2}}}{{ }_{\varepsilon_{1}}^{\infty}}
$$

where the symbols $\stackrel{\infty}{\varepsilon_{1}}$ and $\stackrel{\infty}{\varepsilon_{2}}$ have the obvious meaning.

It is of interest to explore the stresses at points of the family of confocal ellipses which include the boundary,

$$
x_{1}^{2} / a_{1}^{2}+x_{2}^{2} / a_{2}^{2} \leqslant 1
$$

We let $n_{\alpha}$ denote the unit outward normal to the confocal ellipse under consideration and use the notation $\tau_{N}^{*}, \tau_{S}^{*}$ for the normal and tangential stress components, respectively, which are defined by (2.8). The components $n_{\alpha}$ are given by

$$
\begin{aligned}
& n_{1}=x_{1} /\left(a_{1}^{2}+k\right) h, \\
& n_{2}=x_{2} /\left(a_{2}^{2}+k\right) h,
\end{aligned}
$$

where $h$ is the positive root of

$$
h^{2}=x_{1}^{2} /\left(a_{1}^{2}+k\right)^{2}+x_{2}^{2} /\left(a_{2}^{2}+k\right)^{2}
$$

Thus, and by (5.11)

$$
\tau_{N}^{*}=\frac{-A a_{1} a_{2}}{\sqrt{\left(a_{1}^{2}+k\right)\left(a_{2}^{2}+k\right)}}+\sum_{\gamma=1}^{2}\left(A a_{1} a_{2} R_{\gamma} n_{\gamma}^{2}+\stackrel{\infty}{\tau}_{\gamma} n_{\gamma}^{2}\right)
$$

The stress $\tau_{S}^{*}$ is easily found from the relation $\tau_{N}^{*}+\tau_{S}^{*}=\theta^{*}=$ constant.

In order to present the computational results, we must specify the semiaxes ratio $a_{2} / a_{1}$, which follows as a result of (5.13) with given Poisson's ratio $\nu$, internal pressure $P$, and the ratio of the applied load $\tau_{2} / \tau_{1}$. We consider three cases: a traction-free void, a pressurized void, and a rigid inclusion. The ratio $\stackrel{\infty}{\tau}_{2} / \stackrel{\infty}{\tau}_{1}$ is assumed to be 4 for all cases. For the second case, the pressure is prescribed to be $\stackrel{\infty}{\tau}_{1} / 2$, and Poisson's ratio is taken to be 0.15 for the matrix. The corresponding normal stresses on a selection of confocal ellipses are illustrated in Fig. 1-3. 
In Fig. 1, it is clear that the normal stress $\tau_{N}^{*}$ is equal to 0 on the surface of the void $(k=0)$, as required by (2.10). When we move away from the void $k=0$ through ellipses $k>0, \tau_{N}^{*}$ increases toward its limiting value at infinity. Fig. 2 is similar to Fig. 1, but the normal stress on the boundary is $-P$ instead of 0 . We note here that the optimum shapes for different cases, though ellipses, are not proportioned the same, as can easily be seen from (5.13). Concerning Fig. 3, we observe that $\tau_{N}^{*}$ decreases toward its assigned value when $k$ increases. This limiting value (for large $k$ ), as in the case of the void, represents the load at infinity. The tangential stresses for each of these cases are shown in Figs. 4-6.

Acknowledgment. We gratefully acknowledge the support provided by the University of Houston through the Office of Sponsored Projects.

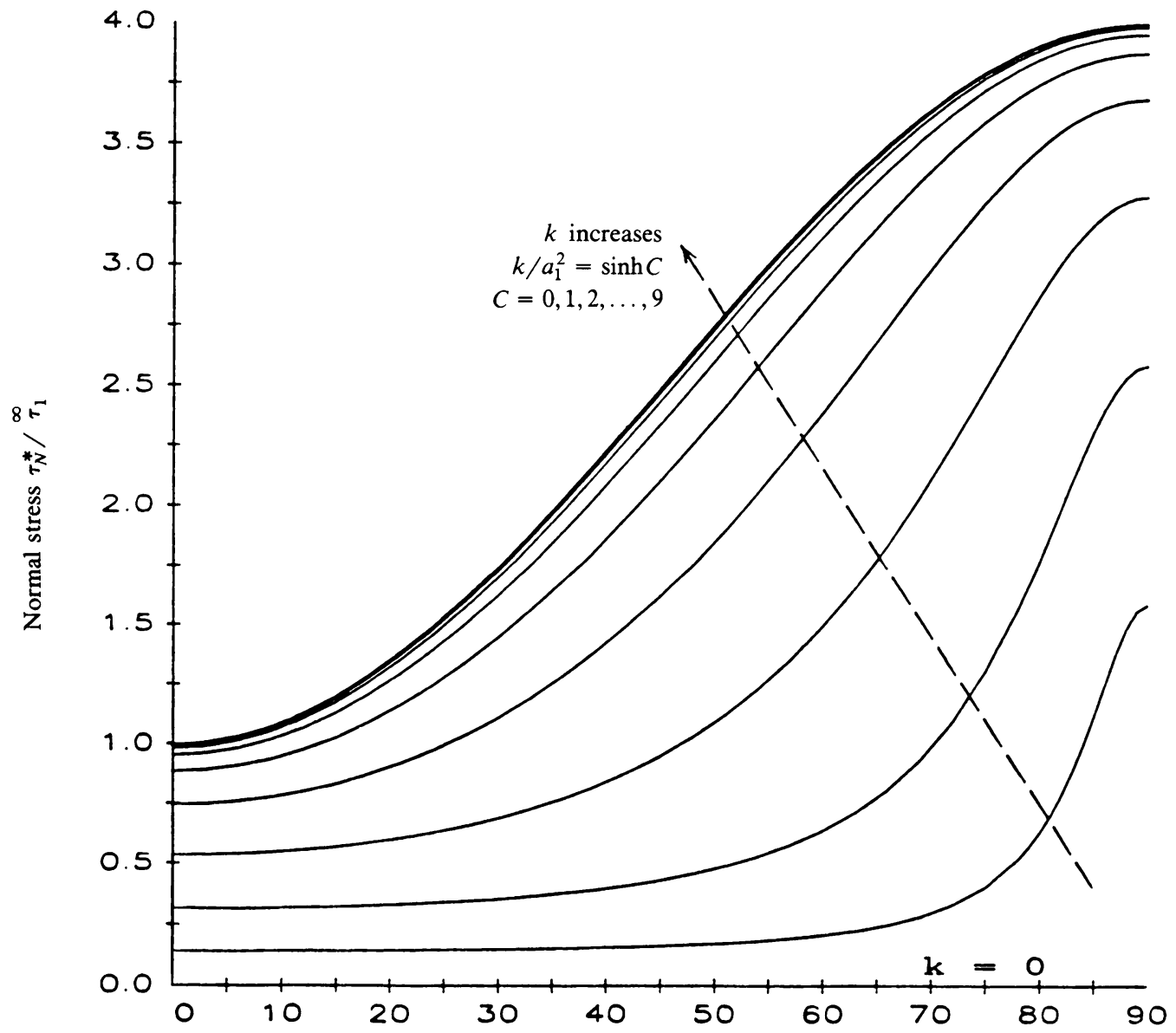

Polar angle $v$ (degrees)

FIG. 1. Normal stresses on confocal ellipses outside the traction-free void 


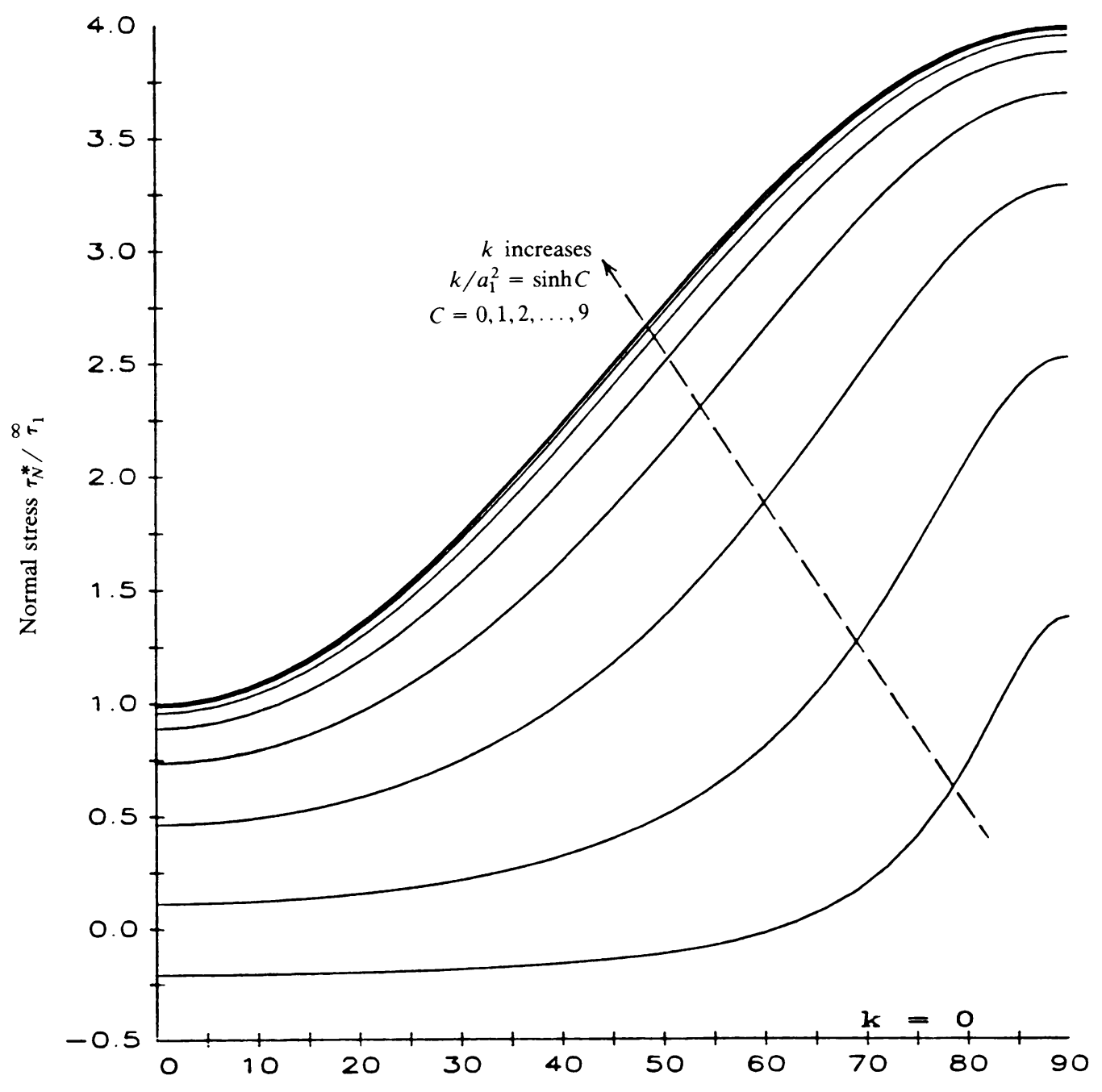

Polar angle $v$ (degrees)

FIG. 2. Normal stresses on confocal ellipses outside the pressurized void 


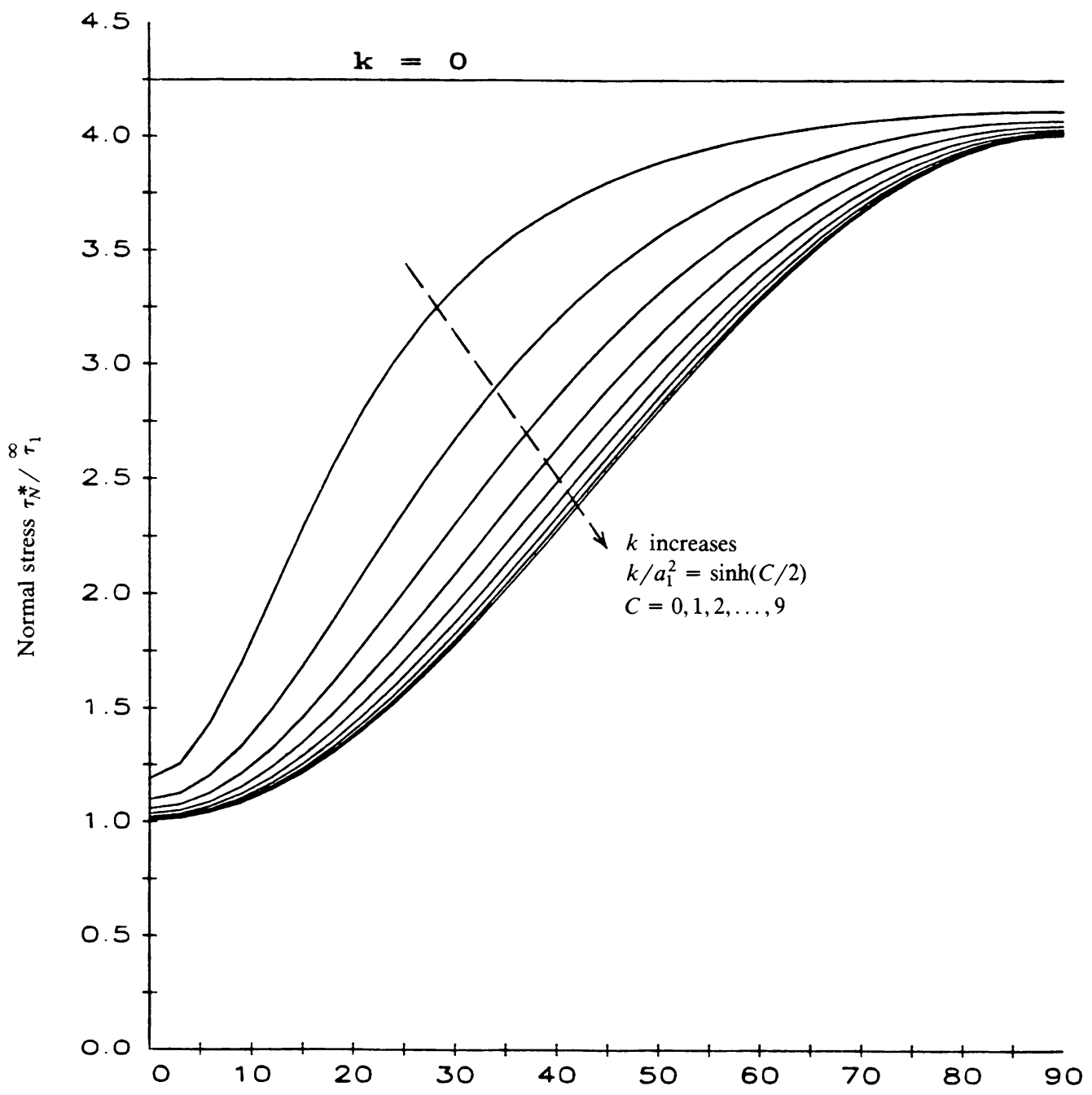

Polar angle $v$ (degrees)

FIG. 3. Normal stresses on confocal ellipses outside the rigid inclusion 


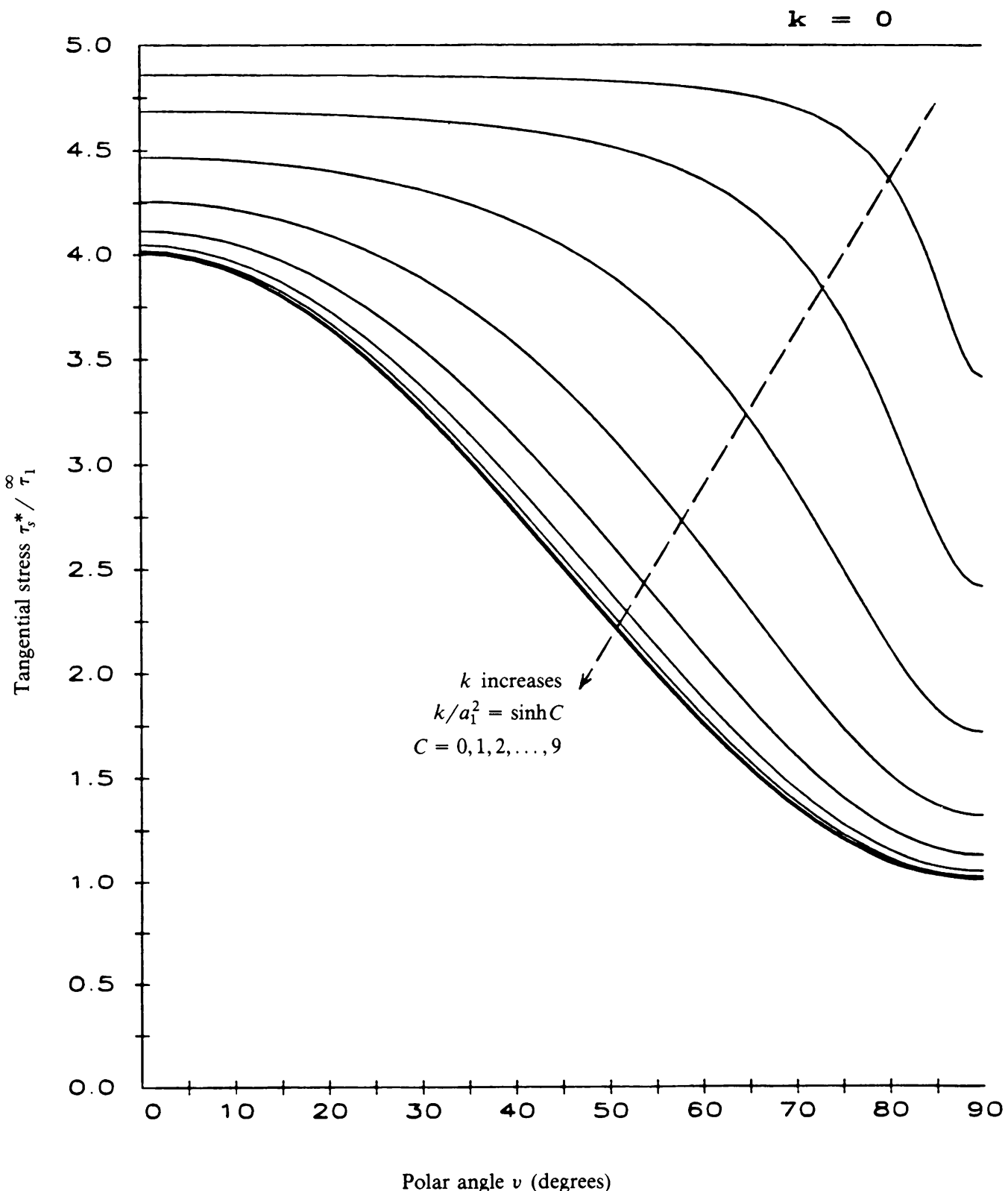

Fig. 4. Tangential stresses on confocal ellipses outside the traction-free void 


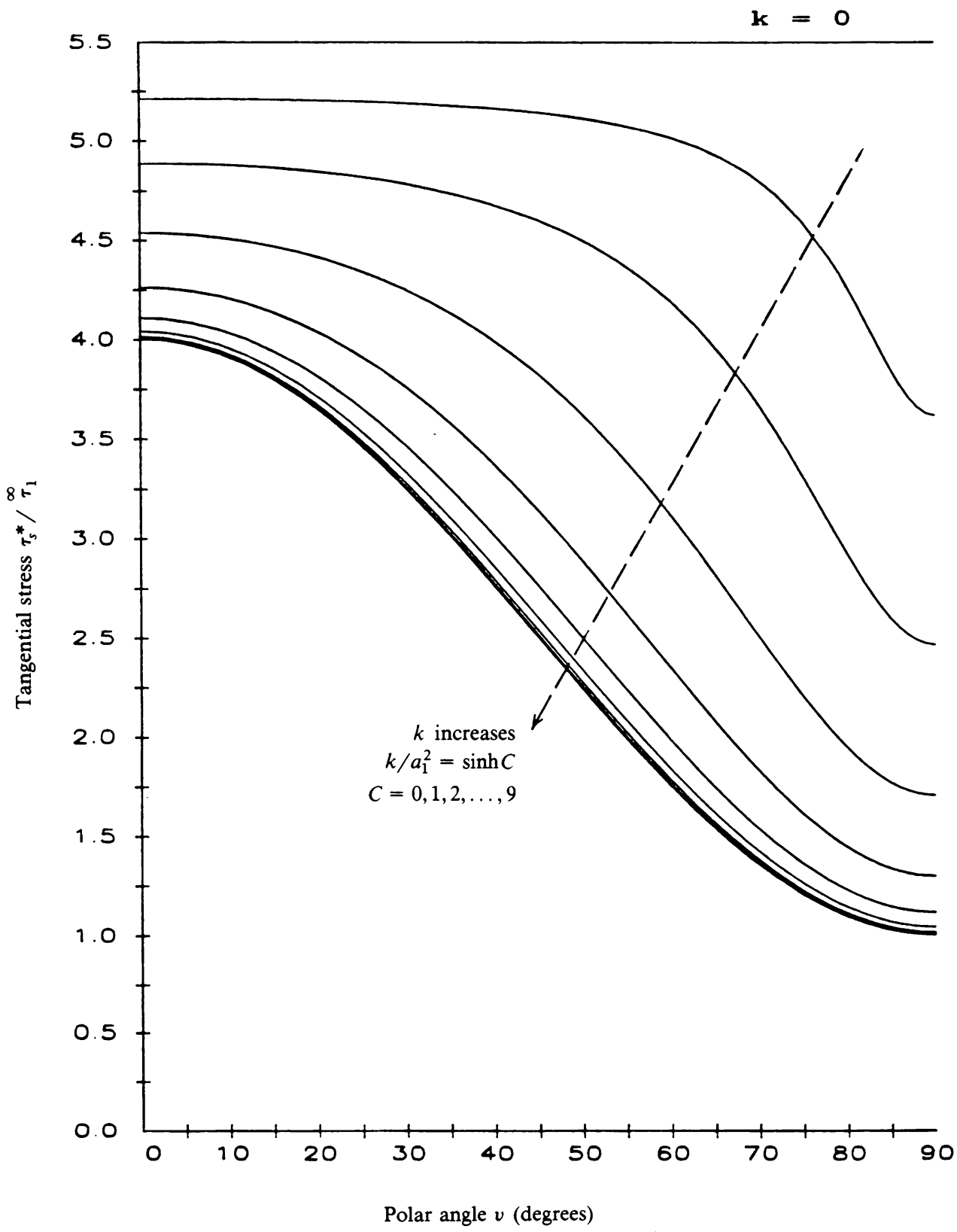

FIG. 5. Tangential stresses on confocal ellipses outside the pressurized void 


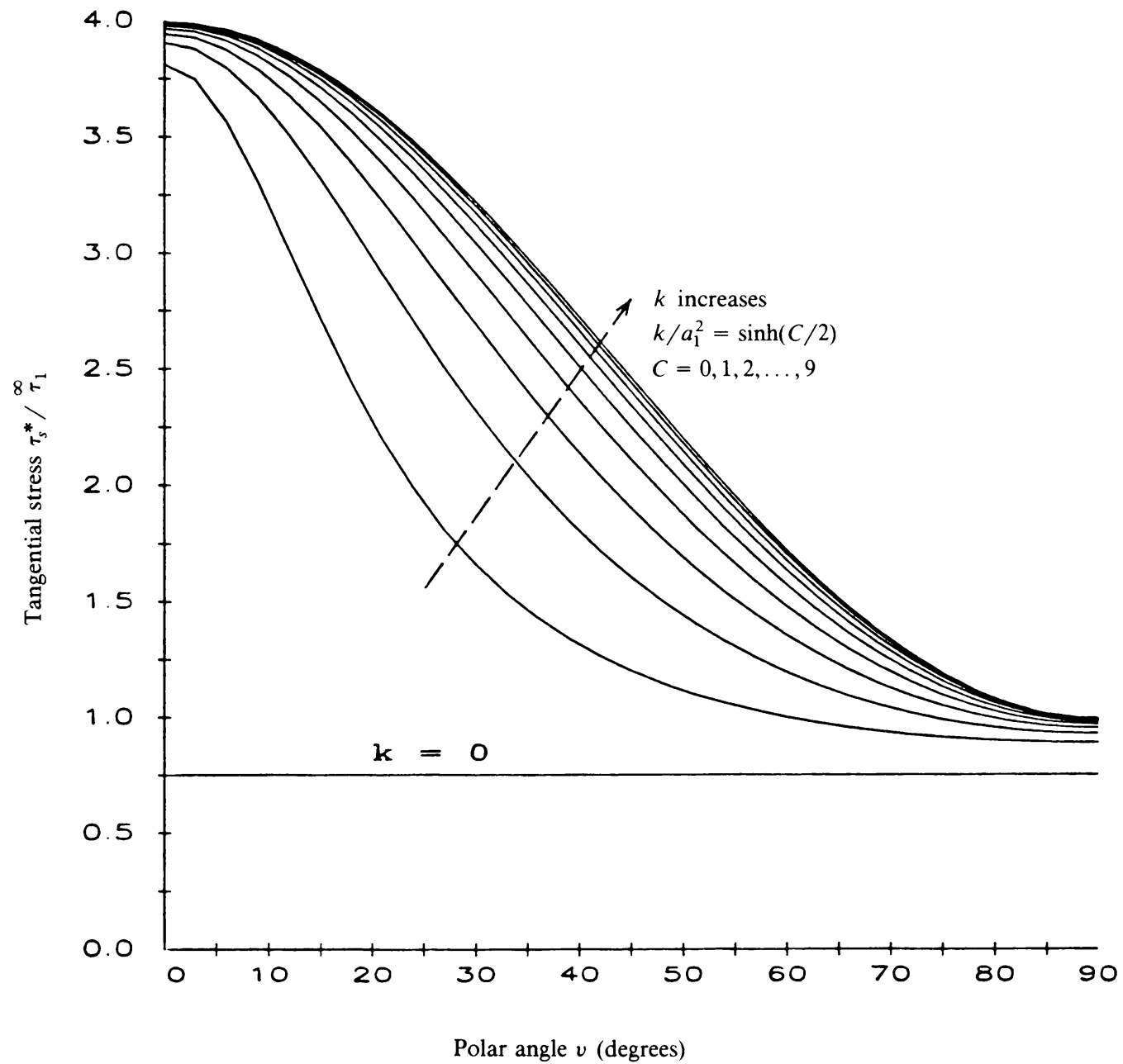

FIG. 6. Tangential stresses on confocal ellipses outside the rigid inclusion 


\section{REFERENCES}

[1] L. T. Wheeler, The problem of minimizing stress concentration at a rigid inclusion, J. Appl. Mech. 52, 83-87 (1985)

[2] B. H. Eldiwany, and L. T. Wheeler, On rigid inclusions of minimum stress concentration, J. Mech. Phys. Solids 34, No. 1, 19-38 (1986)

[3] L. T. Wheeler, On the pole of constant stress surfaces in the problem of minimizing elastic stress concentration, Internat. J. Solids and Structures 12, 779-789 (1976)

[4] L. T. Wheeler, On optimum profiles for the minimization of elastic stress concentration, ZAMM, T235-T236 (1978)

[5] L. T. Wheeler and I. A. Kunin, On voids of minimum stress concentration, Internat. J. Solids and Structures 18, 85-89 (1982)

[6] N. V. Banichuk, Optimality conditions in the problem of seeking the hole shapes in plastic bodies, PMM 41, No. 5. 920-925 (1977)

[7] B. H. Eldiwany and L. T. Wheeler, Groove-bottom contours of minimum stress concentration for antiplane deformation, J. Appl. Mech. 52, No. 2, 379-384 (1985)

[8] L. T. Wheeler, T. E. Tezduyar, and B. H. Eldiwany, Profiles of minimum stress concentration for antiplane deformation of an elastic solid, J. Elast. 15, 271-282 (1985)

[9] A. J. Durrelli, and W. M. Murray, Stress distribution around an elliptic discontinuity in any two-dimensional, uniform, and axial system of combined stress, Exp. Stress Anal. Proc. 1, No. 1, 19-31 (1943)

[10] H. Neuber. Der zugheanspruchte Flachstab mit Optimalem Querschnittubeigang. Forsch. Ingenieurwesen 35. No. 1, 29-30 (1969)

[11] H. Neuber, Zur Optimierung der Spannungskonzentration, in Continuum Mechanics and Related Problems of Analysis. Nauka, Moscow, 375-380 (1972)

[12] G. P. Cherepanov, Inverse problem of the plane theory of elasticity, PMM, 38, No. 6, 963-979 (1974)

[13] G. S. Bjorkman, Jr. and R. Richards, Jr., Harmonic holes-an inverse problem in elasticit?, J. Appl. Mech. 43. No. 3, 414-418 (1976)

[14] G. S. Bjorkman, Jr. and R. Richards, Jr., Optimum shapes for unlined tunnels and cavities, Engineering Geol. 12. $171-179(1978)$

[15] G. S. Bjorkman, Jr. and R. Richards, Jr., Harmonic holes for nonconstant fields, J. Appl. Mech. 46, No. 3 , 573-576 (1979)

[16] G. S. Bjorkman, Jr. and R. Richards, Jr., Harmonic Shapes and Optimum Design, Journal of the Engineering Mechanics Division, ASCE, Vol. 106, pp. 1125-1134, 1980

[17] G. S. Bjorkman, Jr. and R. Richards, Jr., Neutral Holes; Theory and Design, Journal of the Engineering Mechanics Division, ASCE, Vol. 108, pp. 945-960, 1982

[18] B. H. Eldiwany, L. T. Wheeler, A three-dimensional inverse problem for inhomogeneities in elastic solids, J. Elast. In press

[19] Arthur P. Boresi, and Omar M. Sidebottom, Advanced mechanics of Materials, Fourth Ed., John Wiley and Sons, New York, 1985

[20] O. D. Kellogg, Foundations of potential theory, Springer-Verlag, 1929

[21] W. D. McMillan, The theory of the potential, Dover, New York, 1958 